\title{
REVISIÓN
}

\section{Biotratamiento de contaminantes de origen inorgánico $^{(\bullet)}$}

\author{
F.J. Alguacil $^{(*)}$ e Y. Merino ${ }^{(*)}$
}

\begin{abstract}
Resumen De una forma general se asocia el tratamiento biológico de residuos con la eliminación de contaminantes de naturaleza orgánica. Sin embargo, existe cada vez un mayor grado de información, por su importancia, sobre la aplicación de sistemas biológicos en el tratamiento de suelos, sedimentos y aguas contaminadas con componentes inorgánicos tales como metales, radionucleidos y aniones (nitratos y cianuros). Estos componentes pueden ser tóxicos, tanto para los seres humanos como para los organismos empleados para eliminarlos. A diferencia de los contaminantes orgánicos, la mayoría de los inorgánicos no pueden ser degradados aunque se puede alterar su transporte de una localización a otra. Existen varios procedimientos para este tipo de biotratamiento: inmovilización, movilización y transformación de los contaminantes por métodos de bioacumulación, bioadsorción, oxidación y reducción, metilación y demetilación, complejación, degradación y fitoeliminación. En este trabajo se describen estos procesos.
\end{abstract}

Palabras clave: Componentes inorgánicos. Residuos. Biotratamiento.

\section{Bioremediation of inorganic contaminants}

\begin{abstract}
Bioremediation is usually associated with the remediation of organic contaminants. However, there is an increasing amount of information on the application of biological systems to bioremediation of soils, sediments and water contaminated with inorganic compounds which includes metals, radionuclides and anions (e.g. nitrates and cyanides). These compounds can be toxic both to humans and to the organisms used to remediate these toxic components. In contrast to organic compounds, most inorganic contaminants cannot be degraded, but must be remediated by altering their transport properties. Immobilization, mobilization or transformation of inorganic contaminants via bioaccumulation, biosorption, oxidation and reduction, methylation, demethylation, complexation, ligand degradation and phytoremediation are some of the different processes applied in this type of byoremediation. This paper describes these processes.
\end{abstract}

Keywords: Inorganic contaminants. Wastes. Bioremediation.

\section{INTRODUCCIÓN}

Los metales son necesarios para la vida y se utilizan como micronutrientes para los seres humanos, las plantas y los microorganismos. Sin embargo, cuando la concentración de estos micronutrientes en el medio ambiente empieza a alcanzar unos límites superiores a los necesarios para la vida empiezan a ser tóxicos y, por lo tanto, empiezan a ser considerados como contaminantes. La consecuencia

$(\bullet \quad$ Trabajo recibido el día 9 de enero de 1998 y aceptado en su forma final el 9 de junio de 1998.

(*) Centro Nacional de Investigaciones Metalúrgicas, CENIM (CSIC). Av. de Gregorio del Amo, 8. 28040Madrid (España). de este hecho es que, los suelos, aguas, etc., que se han contaminado se convierten en sistemas peligrosos para el hombre y los procesos ecológicos.

Los procesos biológicos se pueden emplear de forma efectiva para eliminar este tipo de materiales contaminantes, por ejemplo metales, radionucleidos $\mathrm{y}$ aniones. Esto es posible porque ciertos componentes biológicos (microorganismos y plantas) pueden modificar en su medio ambiente el carácter tóxico de estos componentes inorgánicos, presentes en una concentración elevada, desde tóxicos a no tóxicos presumiblemente como un mecanismo de defensa. Estos organismos y los procesos que catalizan se pueden utilizar en el biotratamiento de suelos, aguas y efluentes contaminados con dichos componentes inorgánicos. 
Los mecanismos empleados para reducir la toxicidad incluyen la inmovilización, la movilización y transformación de los mismos. Algunos ejemplos son la bioacumulación, la bioadsorción, la oxidación y la reducción, la metilación y la demetilación, complejación, degradación de ligandos y la fitoeliminación. El entendimiento de cada una de estas opciones biológicas hace necesario el conocimiento de cómo los contaminantes inorgánicos interaccionan con los sistemas biológicos y cómo estos se adaptan y responden a estos contaminantes. La adaptación al medio y la presencia de contaminantes inorgánicos (por ejemplo cambiando los niveles de los contaminantes y los valores de $\mathrm{pH}$, temperatura, concentración salina y fuerza iónica) pueden ser variables que favorezcan el uso de sistemas bióticos en el tratamiento de componentes inorgánicos (1). Sin embargo, los cambios iniciales en las concentraciones de los contaminantes inorgánicos y orgánicos y en el $\mathrm{pH}$ o el potencial pueden afectar de forma negativa al proceso, aunque la resistencia a estos cambios suele mejorar con el paso del tiempo (2).

\section{BIOACUMULACIÓN Y BIOADSORCIÓN}

Los microorganismos asimilan y concentran nutrientes catiónicos presentes en medios acuosos y emplean estos mismos mecanismos para la concentración de metales y radionucleidos. El proceso de bioacumulación tiene lugar cuando la energía metabólica se emplea para la asimilación de los componentes inorgánicos. La asimilación que no requiere esta energía se refiere al proceso conocido como bioadsorción, proceso que generalmente requiere de la complejación de los elementos inorgánicos, por ligandos o grupos funcionales, de la pared exterior de la célula. Cuando por el contrario, los microorganismos retienen internamente estos metales es por complejación con diversos ligandos citoplasmáticos como los polifosfatos o las proteínas.

Las posibilidades de este tipo de procesos sobre un gran número de cationes y radionucleidos utilizando bacterias, hongos y algas se han puesto de manifiesto frecuentemente (3). La tabla I muestra algunos de estos microorganismos capaces de

TABLA I.- Microorganismos para la bioadsorción de metales

TABLE I.-Microorganisms for metal biosorption

\begin{tabular}{|l|l|}
\hline \multicolumn{1}{|c|}{ Microorganismo } & \multicolumn{1}{c|}{ Metal, $\mathrm{kg} / \mathrm{kg}$ biomasa } \\
\hline Bacterias & \\
Bacillus sp. & $\mathrm{U}, \mathrm{Cu}, \mathrm{Zn}, \mathrm{Cd}(0,01)$ \\
Pseudomonas aeruginosa & $\mathrm{U}(0,1)$ \\
Pseudomonas putida & $\mathrm{Cd}(0,2)$ \\
Pseudomonas stutzeri & $\mathrm{Zn}$ \\
Pseudomonas cepucia & $\mathrm{Cs}$ \\
Enterobacter cloacae & $\mathrm{Ag}(0,04), \mathrm{Pb}, \mathrm{Cr}, \mathrm{Cu}$ \\
Streptomyces noursei & $\mathrm{As}(0,03)$ \\
Sulfolobus B.C. & $\mathrm{Am}, \mathrm{Pu}, \mathrm{Np}, \mathrm{La}, \mathrm{Th}(0,01)$ \\
Citrobacter & \\
Hongos & $\mathrm{Co}(0,003)$ \\
Neurospora crassa & $\mathrm{U}(0,2), \mathrm{Th}(0,2)$ \\
Rhizopus arrhizus & $\mathrm{Cu}(0,002)$ \\
Penicillum spinulosum & $\mathrm{Cu}, \mathrm{Pb}, \mathrm{Cr}, \mathrm{Zn}, \mathrm{Cd}$ \\
Penicillum chrysogenum & $\mathrm{Cu}(0,002)$ \\
Aspergillus niger & $\mathrm{U}(0,02)$ \\
Aspergillus ansta & $\mathrm{U}(0,001)$ \\
Aspergillus ochraceus & $\mathrm{Cu}, \mathrm{Cr}(0,001)$ \\
Penicillum & $\mathrm{U}$ \\
Tricoderma harzianum & \\
Levadura & $\mathrm{U}(0,1), \mathrm{Cu}(0,8), \mathrm{Co}, \mathrm{Cd}(0,1)$ \\
Sacchanomyces Cerevisiae & \\
Algas & $\mathrm{Cu}(0,03)$ \\
Vaucheria & $\mathrm{Au}(0,1)$ \\
Chlorella vulgaris & $\mathrm{Au}(0,1)$ \\
Spirulina & $\mathrm{Se}(0,001)$ \\
Spirukina platensis & $\mathrm{Cd}(0,7)$ \\
Stichococcus bacillaris & \\
\hline
\end{tabular}


retener elementos metálicos a partir de distintos medios (4). Los microorganismos también pueden ser selectivos con distintos contaminantes inorgánicos y en algunos casos esta selectividad puede ser aún mayor que la presentada por compuestos químicos sintéticos.

Los mecanismos de bioacumulación o bioadsorción suelen estar controlados por la preferencia de los metales por las distintas posiciones de enlace con los ligandos, por ejemplo el $\mathrm{Cd}^{2+}$ puede ser complejado por polifosfatos o por proteínas, presentes en el citoplasma, que contienen azufre, el metal puede precipitarse en el exterior de la célula como un fosfato de cadmio o como un sulfuro de cadmio. Esta precipitación tiene lugar porque el fósforo y el azufre son ligandos afines por el $\mathrm{Cd}^{2+}$ que es un ácido débil.

Además de las paredes celulares, otros materiales extracelulares pueden dar lugar a la bioadsorción de metales. Algunos microorganismos producen polímeros en el exterior de la célula. Estos exopolímeros pueden adsorber metales o radionucleidos de distintos medios (5) y tienen una natura-

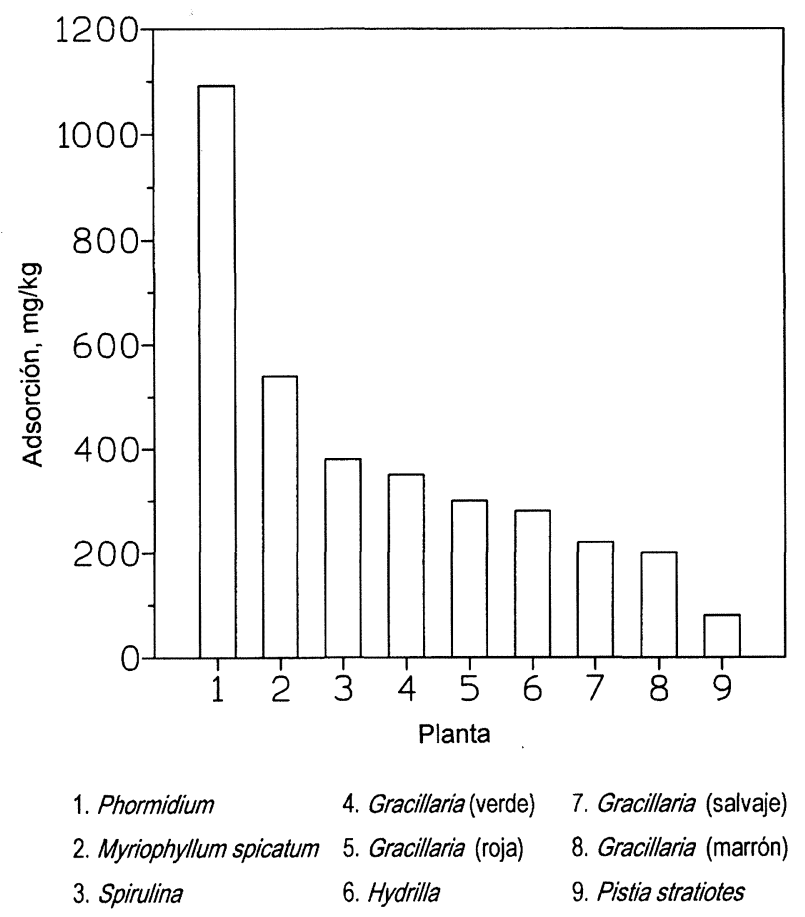

FIG. 1. - Adsorción específica de cadmio en distintas biomasas. Concentración inicial de cadmio: $1 \mathrm{mg} / \mathrm{L}$. Densidad de biomasa: $0,01 \mathrm{~kg} / \mathrm{L}$ (base húmeda) excepto en el caso de Phormidium (94 mg/L, base seca).

FIG. 1.- Specific adsorption of cadmium onto different biomass. Initial cadmium concentration: $1 \mathrm{mg} / \mathrm{L}$. Biomass density: $0,01 \mathrm{~kg} / \mathrm{L}$ (wet) except for Phormidium (94 mg/L, dry). leza ácida con distintos grupos funcionales que son los responsables de la coordinación con la especie inorgánica. La producción de los exopolímeros puede ser controlada con las condiciones de cultivo, incluyendo la fuente de obtención de carbono y nitrógeno y la presencia o ausencia de iones nutrientes o contaminantes.

Como se ha mencionado, los metales se pueden inmovilizar mediante la precipitación en la pared celular de determinados compuestos. Además del caso del cadmio; otro elemento altamente tóxico, como el uranio, puede ser precipitado como un fosfato de uranilo mediante el empleo de estos biosistemas generadores de fosfato (6), los cuales se han usado también en el tratamiento de efluentes que contienen otros metales pesados (7).

Las plantas y las algas pueden utilizarse en la eliminación de contaminantes inorgánicos de efluentes (8-12). La efectividad de las distintas especies ensayadas (microalgas, macroalgas y plantas) es muy distinta en la eliminación de cinc y cadmio a partir de una disolución acuosa como queda reflejado en las figuras 1 y 2 (13).

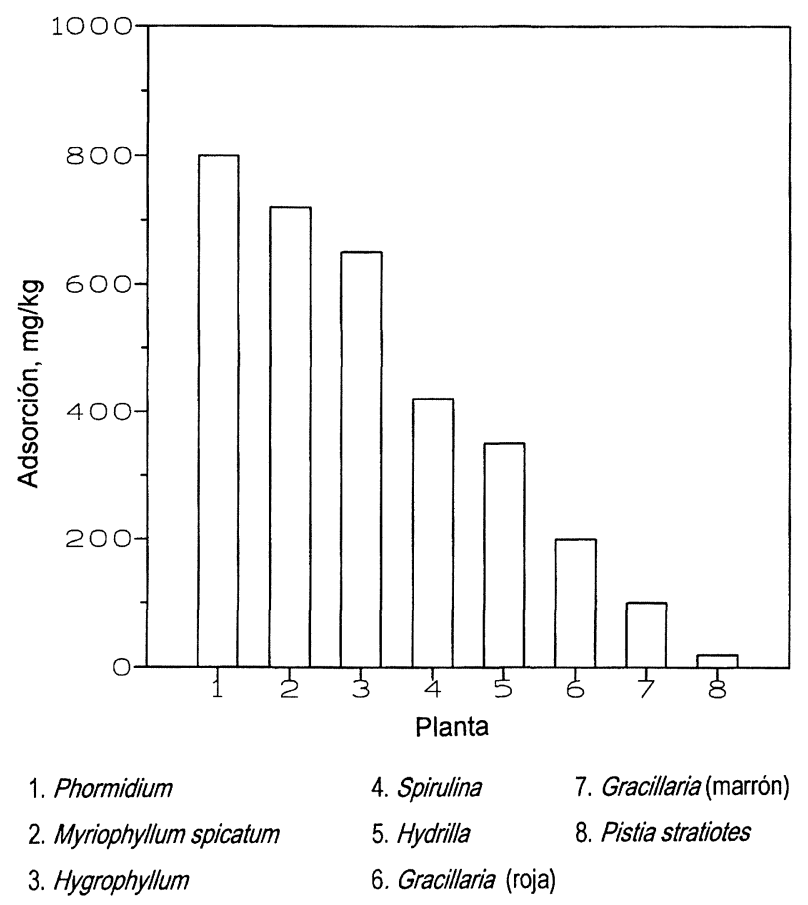

FIG. 2.- Adsorción específica de cinc en distintas biomasas. Concentración inicial de cinc: $1 \mathrm{mg} / \mathrm{L}$. Densidad de biomasa: $0,02 \mathrm{~kg} / \mathrm{L}$ (base húmeda) excepto en el caso de Phormidium $(81 \mathrm{mg} / \mathrm{L}$, base seca), Hydrilla $(0,1 \mathrm{~kg} / \mathrm{L})$ y Pistia stratiotes $(0,1 \mathrm{~kg} / \mathrm{L})$.

FIG. 2.- Specific adsorption of zinc onto various biomass. Initial zinc concentration: $1 \mathrm{mg} / \mathrm{L}$. Biomass density: $0,02 \mathrm{~kg} / \mathrm{L}$ (wet) except for Phormidium $(81 \mathrm{mg} / \mathrm{L}$, dry), Hydrilla $(0,1 \mathrm{~kg} / \mathrm{L})$ and Pistia stratiotes $(0,1 \mathrm{~kg} / \mathrm{L})$. 
Mediante el empleo de biomasa inmovilizada también se pueden retener determinados cationes; en algunos casos se hace necesario algún tipo de pretratamiento de esta biomasa para mejorar sus características, bien pensando en su reutilización (14) o para aumentar su capacidad de bioadsorción (15).

En algunos casos la biomasa puede inmovilizarse en un gel, resultando un sistema similar a las resinas de intercambio iónico. La figura 3 muestra cómo un sistema de estas características puede ser efectivo en la biorretención de ciertos elementos metálicos, cinc, cobre y cadmio. El pretratamiento de la biomasa con una disolución alcalina caliente aumenta la capacidad de bioadsorción de estos metales pesados. Como biomasa se empleó levadura (Saccharomyces cerevisiae) ampliamente disponible en la industria alimentaria, la cual se inmovilizó en una matriz obtenida a partir de Macrocystis pyrifera (16).

Otros soportes que pueden utilizarse para la inmovilización son agar, alginatos, celulosa, poliacrilamida, derivados toluénicos del diisocianato y glutaraldehido (5).

Los bioadsorbentes que se pueden obtener de forma comercial emplean algunos de los microorganismos citados, tratados según el fabricante, con el fin de aumentar su capacidad para la retención de metales. Algunos procesos son el Bioclaim que emplea Bacillus sp., el Algasorb que utiliza un alga,

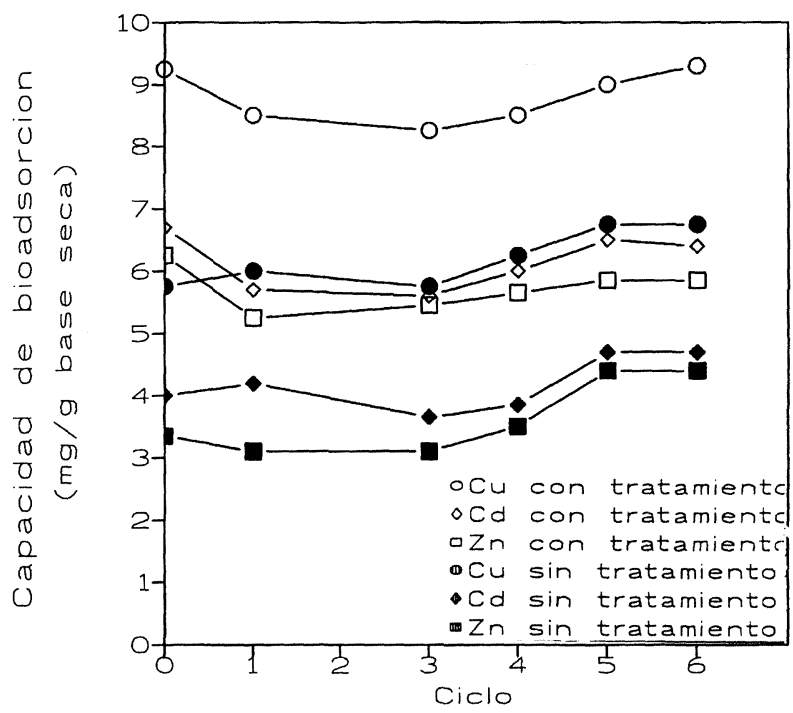

FIG. 3.- Bioadsorción de metales en levadura sin tratamiento o con tratamiento. Concentración inicial de cobre, cinc y cadmio entre 16-18 mg/L, pH 5,0 y $23{ }^{\circ} \mathrm{C}$.

FIG. 3.- Biosorption of metallic ions to immobilized yeast and caustic treated yeast. Initial concentration of copper, zinc and cadmium in the range $16-18 \mathrm{mg} / \mathrm{L}, \mathrm{pH} 5,0$ and $23^{\circ} \mathrm{C}$. el Bio-fix que incluye el tratamiento con varios bioadsorbentes (17) y el B.V.Sorbex que emplea distintos tipos de biomasa microbial (3). Estos sistemas pueden utilizarse bajo diferentes condiciones operacionales (discontinuo, continuo, lecho fijo, etc.), con bajos costes de producción y con un relativo amplio margen de alteración para adecuarse al tratamiento requerido.

Un ejemplo de empleo de sistemas de este tipo para la eliminación de metales $(\mathrm{Cu}$ y $\mathrm{Fe}$ ) es la planta de Homestake Mine (EE.UU.) (1). En esta planta se utilizan contactores giratorios, que contienen Pseudomonas sp., para la eliminación de cobre y hierro de un efluente líquido. Se tratan cerca de $15 \cdot 10^{6} \mathrm{~L} /$ día eliminándose el $95-98 \% \mathrm{Cu}$ y $\mathrm{Fe}$ contenidos en el efluente de entrada a la planta de bioacumulación/bioadsorción.

\section{FITOELIMINACIÓN}

Este término se refiere al uso de plantas para el tratamiento (descontaminación) de suelos y aguas. También se ha empleado este tipo de procesamiento para la eliminación de contaminantes orgánicos (18) fundamentalmente en la rizosfera (19).

La fitoeliminación puede a su vez subdividirse en dos tipos de tratamiento: fitoestabilización y fitoextracción (20). La fitoestabilización emplea plantas para estabilizar suelos contaminados mediante la disminución de la erosión producida por los vientos y por el agua, disminuyendo las filtraciones acuosas y, por lo tanto, disminuyendo la lixiviación de los elementos contaminantes. La fitoextracción se refiere al procedimiento por el cual los contaminantes inorgánicos son separados de las partes de las plantas que están por encima del nivel del suelo. Una vez recolectadas estas, los contaminantes inorgánicos se separan de la biomasa vegetal.

Estos procesos de fitoeliminación ofrecen cada vez un mayor grado de interés (21-27). Las ventajas del fitotratamiento se resumen en bajos costos de inversión, estabilización de los suelos, no se altera el medio ambiente (no hay excavaciones) y se reduce la posibilidad de lixiviación y el transporte de los contaminantes inorgánicos en el suelo. Los mayores costes del proceso se refieren a que es necesario plantar, cultivar, recolectar, almacenar la biomasa contaminada y volver a repetir el proceso. La figura 4 muestra el ciclo de fitoextracción (28).

Sin embargo, el fitotratamiento es en algunos casos muy ventajoso; así, para el tratamiento de suelos contaminados con elementos tóxicos (metales como $\mathrm{As}, \mathrm{Cd}, \mathrm{Cr}_{\text {total }}, \mathrm{Cu}, \mathrm{Hg}$ (inorgánico), $\mathrm{Pb}$, $\mathrm{Zn}$ y radionucleidos: ${ }^{137} \mathrm{Cs},{ }^{239} \mathrm{Pu},{ }^{90} \mathrm{Sr}$ ); este tipo de procesado presenta unos costes del orden de tres veces de magnitud menor que los que se tienen con métodos convencionales de detoxificación (27). 


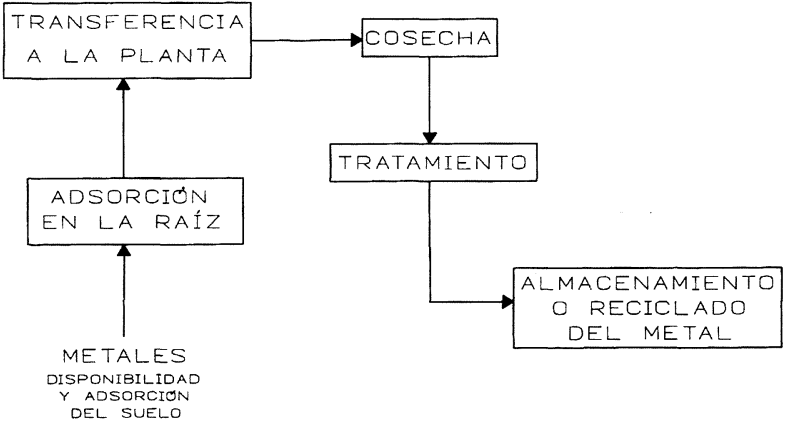

FIG. 4.- Esquema del proceso de fitoextracción.

FIG. 4.- Scheme for phytoextraction.

La tabla II muestra una serie de plantas capaces de retener varios metales (26).

Otra posible limitación del fitotratamiento es que necesariamente la planta debe crecer en el suelo o en el material contaminado. En las localizaciones en las que el contenido de los contaminantes inorgánicos es alto se puede adaptar el crecimiento de las plantas por dilución de estos contaminantes hasta niveles aceptables. Es evidente que la planta sólo puede acumular aquellos elementos tóxicos que pueden alcanzarla a través de las raíces o que son solubles en el agua del subsuelo y pueden ser transportados por procesos de transpiración o movimientos del agua en el subsuelo, por eso, es díficil que los contaminantes localizados por debajo de la raíz puedan ser extraidos por la planta.

\section{METILACIÓN Y DEMETILACIÓN}

Los microorganismos pueden metilar o demetilar los metales. La metilación de estos puede ser un proceso de detoxificación que sirva para proteger a ciertos organismos de los iones metálicos más tóxicos (29). Sin embargo, la metilación en algunos casos puede aumentar el carácter tóxico de los metales hacia los seres humanos, por ejemplo el

TABLA II.- Plantas que pueden hiperacumular distintos metales

TABLE II.- Plants that can hyperaccumulate more than one metal

\begin{tabular}{|l|l|}
\hline \multicolumn{1}{|c|}{ Especie } & Metales acumulados \\
\hline Thlaspi alpestre & plomo, cinc, níquel \\
Viola calaminara & níquel, cinc \\
Thlaspi rotundifolium & plomo, níquel \\
Thlaspi caerulescens & níquel, cinc, cadmio \\
Alyssum montanum & cobre, níquel \\
Haumaniastrum katangese & cobre, cobalto \\
\hline
\end{tabular}

caso del mercurio en forma de $\mathrm{CH}_{3} \mathrm{Hg}$. Además de este elemento, otros metales que se pueden metilar son el arsénico, oro, plomo, paladio, platino, estaño y talio.

Los procesos de metilación conducen generalmente a la formación de compuestos volátiles; así, la metilación del selenio en presencia de bacterias (Pseudonomas fluorescens K27 o Rhodobacter sphaeroides 2.4.1) da lugar, entre otros productos, a la formación de los compuestos volátiles seleniuro de dimetilo y diseleniuro de dimetilo (30).

El arsénico es un elemento muy tóxico para el ser humano por lo que también se ha estudiado este tipo de tratamiento para reducir su presencia en suelos contaminados con él. La lixiviación anaeróbica en presencia de bacterias (por ejemplo Rhodospirillum rubrum) resistentes a la presencia de arseniato y otros oxianiones metálicos, puede dar lugar a la reducción del arseniato. En estas condiciones se puede disolver el $45 \%$ del $\mathrm{As}_{2} \mathrm{~S}_{3}$ inicialmente presente en el suelo contaminado ( $8,3 \mathrm{~g} \mathrm{As} / \mathrm{kg}$ material), convirtiendo Methanobacterium thermoautotrophicum el 25\% del arsénico presente en el medio a especies volátiles (31). Un aspecto muy importante, desde el punto de vista de ingeniería del proceso, es que toda eliminación del arsénico como especie volátil conlleva la necesidad de fijación de este elemento tóxico a partir del gas producido.

Los procesos de demetilación dan lugar a la ruptura de los enlaces metal-metilo y a la formación de especies metálicas cargadas. Así, el $\mathrm{CH}_{3} \mathrm{Hg}$ puede degradarse por la liasa organomercurial o por otro promotor del proceso para formar $\mathrm{CH}_{4} \mathrm{y} \mathrm{Hg}^{2+}$; el $\mathrm{Hg}^{2+}$ puede reducirse a $\mathrm{Hg}^{\circ}$ que se volatiliza (29 y 32 ).

\section{COMPLEJACIÓN}

Ciertos microorganismos pueden formar agentes complejantes o ligandos que pueden aumentar la solubilidad y movilidad de los metales en el medio. La complejación de los radionucleidos y/o de los elementos metálicos tóxicos puede ser un mecanismo para la detoxificación ya que reducen la concentración, en el medio acuoso, del elemento contaminante libre.

Entre los elementos o compuestos que pueden dar lugar a este tipo de reacciones se encuentran el hierro, $\mathrm{PuO}_{2}$, torio y uranio, mientras que los organismos que producen estos agentes complejantes incluyen Anabea sp., Pseudonomas aeruginosa y Cylindrocarpon $s p$.

En este sentido, una bacteria marina (Vibrio alginolyticus) produce proteínas extracelurares que pueden complejar al cobre y detoxificar, por lo tanto, los medios que lo contienen (33 y 34 ). 


\section{DEGRADACIÓN DE LIGANDOS}

Como se ha mencionado, la formación de complejos metálicos puede ser un medio para su eliminación del sustrato que los contiene. También la degradación de ligandos puede ser un medio para reducir la movilidad de los metales y en algún caso, como es el del ion cianuro, para reducir la concentración de este anión extremadamente tóxico.

El AEDT es un agente tipo quelato que tiene una serie de usos industriales y que puede aumentar la solubilidad y movilidad de los metales en el medio ambiente. Este agente puede ser degradado por asociaciones de bacterias o por alguna de ellas de forma individual (35). En algunos casos y empleando la cepa BNC1, la degradación ha sido una función del tipo de metal unido al AEDT (36); el AEDT unido al $\mathrm{Co}(\mathrm{II})$ y (III) o al $\mathrm{Ni}$ (II) no se degrada (37), sin embargo, la unión con otros metales base $(\mathrm{Cu}$ y $\mathrm{Zn}$ ) no dificulta su degradación. Una posibilidad para el biotratamiento de materiales contaminados sería entonces el empleo del AEDT para, a continuación degradar, en biorreactores, los complejos AEDT-metal.

La presencia de cianuro en distintos efluentes requiere de su eliminación. Algunos microorganismos pueden degradar tanto el cianuro libre como el complejado con determinados metales ( 38 y 39 ).

Determinados microorganismos aerobios pueden degradar el $\mathrm{CN}^{-}(40)$, la degradación puede acelerarse si el medio es pretratado con el reactivo de Fenton $\left(\mathrm{H}_{2} \mathrm{O}_{2}, \mathrm{Fe}^{2+}\right)$ (41); sin embargo, el complejo $\mathrm{Fe}(\mathrm{CN})_{6}^{4-}$ es estable a este tratamiento (42).

Ciertas bacterias anaerobias pueden degradar tanto el cianuro como el acetonitrilo (43); las reacciones responsables de esta descomposición son:

a) Cianuro:

$$
\begin{gathered}
\mathrm{HCN}+2 \mathrm{H}_{2} \mathrm{O} \rightarrow \mathrm{HCOO}^{-}+\mathrm{NH}_{4}^{+} \\
4 \mathrm{HCOO}^{-}+4 \mathrm{H}^{+} \rightarrow \mathrm{CH}_{4}+3 \mathrm{CO}_{2}+2 \mathrm{H}_{2} \mathrm{O}
\end{gathered}
$$

b) Acetonitrilo:

$$
\begin{gathered}
\mathrm{CH}_{3} \mathrm{CN}+2 \mathrm{H}_{2} \mathrm{O} \rightarrow \mathrm{CH}_{3} \mathrm{COO}^{-}+\mathrm{NH}_{4}^{+} \\
\mathrm{CH}_{3} \mathrm{COO}^{-}+\mathrm{H}^{+} \rightarrow \mathrm{CH}_{4}+\mathrm{CO}_{2}
\end{gathered}
$$

En la ya mencionada planta de Homestake Mine se degrada el $\mathrm{CN}^{-}$de un determinado efluente a razón de $15 \cdot 10^{6} \mathrm{~L} /$ día (1). Los contactores biológicos, cargados con Pseudomonas sp., tratan el efluente que contiene $4 \mathrm{mg} \mathrm{CN}^{-} / \mathrm{L}$ para reducir la concentración de este elemento tóxico hasta 0,06 $\mathrm{mg} / \mathrm{L}$.

\section{OXIDACIÓN Y REDUCCIÓN}

La influencia que ciertos microorganismos tienen en la solubilidad y/o movilidad de determinados contaminantes inorgánicos se debe a procesos de oxidación (captando electrones y aumentando la valencia del metal) o de reducción (cediendo electrones y, por lo tanto, disminuyendo la valencia del elemento metálico). Como es sabido, la solubilidad y toxicidad de los metales cambia con su valencia, por ejemplo, el $\mathrm{Cr}$ (III) es un elemento que respecto al medio ambiente no es tóxico y presenta una movilidad discreta, sin embargo, el $\mathrm{Cr}(\mathrm{VI})$ es un elemento muy tóxico y móvil.

Los procesos enzimáticos de oxidación-reducción alteran de forma significativa la solubilidad de elementos como, cromo, hierro, manganeso, mercurio, nitrógeno (nitrato), selenio y uranio (44). Entre los procesos indirectos que pueden eliminar contaminantes metálicos se incluye la precipitación de sulfuros metálicos.

\subsection{Procesos directos: reducción enzimática}

Diversas bacterias pueden reducir elementos metálicos (Fe(III) o Mn(IV)) adaptando la oxidación de materiales orgánicos o hidrógeno al proceso (44). Así, en medio anaerobio y en ausencia de sulfatos, estas bacterias pueden reducir al Fe(III) y aumentar su movilidad cuando está contenido en minerales oxidados; también pueden ser útiles en la oxidación de materiales orgánicos no fermentables y en la degradación de ciertos contaminantes aromáticos.

Las bacterias también pueden ayudar a la solubilización del óxido de plutonio(IV) en presencia de nitrilotriacetato (NTA) (45). El Pu(IV) insoluble se reduce a $\mathrm{Pu}(\mathrm{III})$ soluble, que se compleja con el NTA. El NTA a su vez oxida el Pu(III) a Pu(IV) dando como producto final el complejo de Pu(IV)NTA, que es soluble; el proceso puede, por tanto, solubilizar y eliminar el plutonio de suelos o sedimentos contaminados por este elemento.

El mercurio puede eliminarse mediante el empleo de bacterias aerobias, que reducen el $\mathrm{Hg}$ (II) soluble a $\mathrm{Hg}^{\circ}$ volátil (46 y 47).

El selenio es un elemento tóxico cuya presencia es un peligro permanente para la vida y la agricultura; este metal es sumamente peligroso como seleniato (Se(VI)) que además es un anión con una gran movilidad. Una forma de biotratamiento de materiales contaminados en este anión es mediante reducción microbiana del mismo a selenito (Se(IV)) $\mathrm{y}$, posteriormente, a selenio metal, elemento que se fija, no es tóxico y aparece normalmente como un precipitado rojo (48-50). 
El nitrato también es un anión a eliminar en aquellos medios contaminados por él (51). Una serie de microorganismos pueden utilizar el $\mathrm{NO}_{3}^{-}$ como un aceptor terminal de electrones, que ayuda al crecimiento microbial transformándolo en $\mathrm{N}_{2}$. Estos procesos se conocen con el nombre de bidenitrificación. En algunos casos, la eliminación de este anión pasa por un proceso de nitrificación $\left(\mathrm{NH}_{4}^{+} \mathrm{a}\right.$ $\mathrm{NO}_{3}^{-}$) y luego de denitrificación $\left(\mathrm{NO}_{3}^{-}\right.$a $\left.\mathrm{N}_{2}\right)(52)$. Estos procedimientos de biodenitrificación también han tenido éxito en el tratamiento de aguas contaminadas, con nitrato, procedentes del procesado de minerales uraníferos (53).

\subsection{Procesos de reducción indirecta}

Las bacterias, mediante procesos indirectos, pueden eliminar los elementos metálicos de los efluentes líquidos que los contienen. El sulfuro, formado por la reducción de los sulfatos, reacciona con elementos como la plata, cadmio, cerio, galio, níquel, plomo, selenio, mercurio, cinc y estroncio, para formar sulfuros metálicos insolubles. Estos precipitan y se pueden separar sin muchas dificultades del medio acuoso por varios procedimientos: sedimentación, centrifugación o filtración.

Las mayores aplicaciones de este procedimiento se encuentran en el tratamiento de aguas de mina (54 y 55). La eliminación de estos contaminantes inorgánicos es efectiva mientras que las bacterias y las condiciones experimentales den lugar a la reducción del sulfato (56).

La figura 5 muestra cómo evoluciona la eliminación de tres elementos contaminantes (cinc, manga-

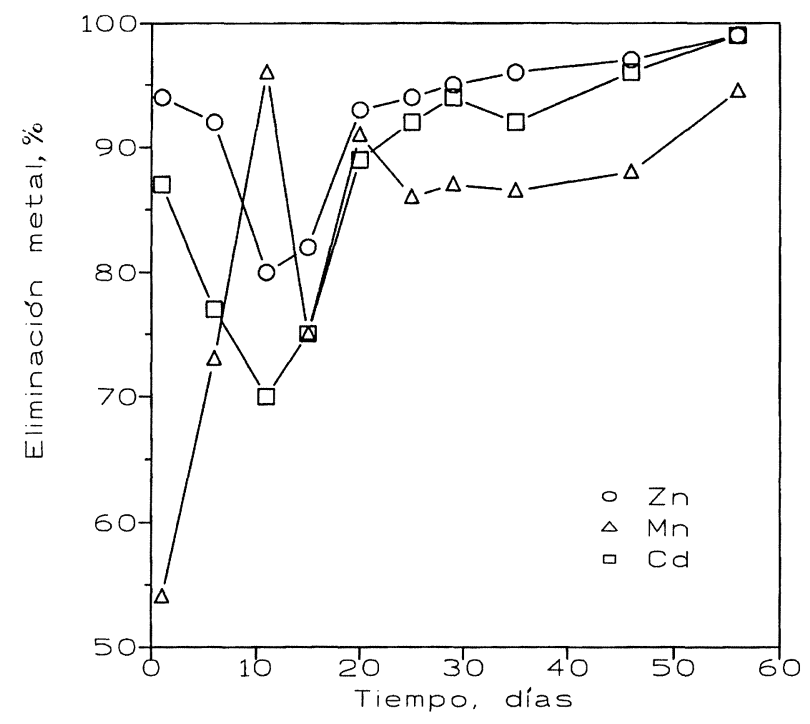

Fig. 5.- Eliminación de metales en biorreactores.

FIG. 5.- Remediation of selected metallic elements in bioreactors. neso y cadmio) presentes en aguas de mina. La operación se llevó a cabo en un biorreactor empleando un sistema inoculado con bacterias reductoras de sulfato (SRB) (57).

\subsection{Procesos de oxidación}

Ciertos microorganismos pueden obtener la energía de la oxidación de compuestos inorgánicos reducidos, por ejemplo hierro y azufre. Así, Thiobacillus ferrooxidans obtiene la energía de la oxidación de minerales sulfurados y del Fe(II). La oxidación produce ácido sulfúrico, que puede solubilizar a una serie de elementos metálicos, como, por ejemplo plata, arsénico, cadmio, mercurio, uranio (58-60).

El proceso conocido como biolixiviación, aplicado a la recuperación de metales en procesos metalúrgicos comerciales, puede ser también una solución a la bioeliminación de contaminantes inorgánicos presentes en determinadas localizaciones.

\section{CONCLUSIÓN}

Los procesos de biotratamiento encaminados a la eliminación de contaminantes inorgánicos, presentes en el medio ambiente, tienen buenas perspectivas de aplicación.

Casi todos se caracterizan porque son tecnologías de tratamiento in situ, presentando una serie de ventajas: no necesitan de la extracción del material contaminado, no se inmoviliza o destruye de manera apreciable el medio ambiente afectado, etc.; sin embargo, en muchos casos, deben ser procesos capaces de actuar en las condiciones hidrogeológicas existentes en el lugar de aplicación del biotratamiento lo que da lugar a que los procesos sean menos fácilmente controlables que los considerados como no in situ. Además, en algunos casos, son procesos de detoxificación a largo plazo (fitoeliminación), con lo que alguna de sus ventajas, por ejemplo las económicas, pueden verse afectadas o contrarrestadas por la necesidad de eliminación rápida de los contaminantes.

Cuando los elementos tóxicos son varios, es necesario emplear un tratamiento múltiple y utilizar una serie de procedimientos que no favorezcan la movilidad o la toxicidad de uno o algunos de los contaminantes cuando se trata de inertizar o destruir otros.

La evaluación de la aplicación de biotratamiento in situ y la elección de las tecnologías necesarias para la solución de problemas de contaminación medioambiental necesitan, por tanto, de la consideración de diversos factores, y su aplicación debe ser 
también comparada, en lo posible, con otras tecnologías existentes buscando la elección de la estrategia correcta en cada caso particular.

\section{Agradecimiento}

Al CSIC por la ayuda prestada para la realización de este trabajo.

\section{REFERENCIAS}

(1) WhitLock, J.L. Geomicrobiol. J., 8, 1990: 241-249.

(2) Bolton Jr., H. y Gorby, Y.A. Bioremediation of Inorganics. R.E. Hinchee, J.L. Means y D.R. Burris (Eds.), Battelle Press. Columbus (Ohio), 1995.

(3) Volesky, B. Biosorption of Heavy Metals, CRC Press, Boca Raton (Florida), 1990.

(4) Fujita, T., Kogita, H., Mamiya, M. y Yen, W.-T. Proc. of the XIX International Mineral Processing Congress, Vol.4, SME, Littleton (New Hampshire), 1995.

(5) Gadd, G.M. y White, C. TIB Tech., 11, 1993: 353-359.

(6) Macaskie, L.E., Empson, R.M., Cheetham, A.K., Grey, C.P. y Skarnulis, A.J. Science, 257, 1992: 782-784.

(7) MaCAskie, L.E. J. Chem. Technol. Biotech., 49, 1990: 357-364.

(8) Green, B. y Bedell, G.W. Introduction to Applied Phycology. I. Akatsuka (Ed.), Academic Publishing, La Haya, 1990.

(9) Sela, M., Huttermann, A. y Tel-or, E. Physiol. Plant. 79, 1990, 547-553.

(10) Chawla, G., Singh, J. y Viswanathan, P.N. Bul. Environ. Toxicol., 47, 1991: 84-90.

(11) Guven, K.C. Bot. Marina, 35, 1992: 337-340.

(12) Wilde, E.W. y Benemann, J.R. Biotech. Adv., 11, 1993: 781-812.

(13) Wang, T.C., Weissman, J.C., Ramesh, G., VARADARAJAN, R. y BENEMANN, J.R. Bioremediation of Inorganics. R.E.Hinchee, J.L. Means y D.R. Burris (Eds.), Battelle Press. Columbus (Ohio), 1995.

(14) Brierley, C.L. y Brierley, J.A., Biohydrometallurgical Technologies, Vol.2, A.E. Torma, M.L. Apel y C.L. Brierley (Eds.), TMMMS, Wyoming (EE.UU.), 1993.

(15) Brady, D., Stoll, A. y Duncan, J.R. Environ. Technol., 15, 1994, 429-438.

(16) Lu, Y. y WILKINS, E. Bioremediation of Inorganics. R.E. Hinchee, J.L. Means y D.R. Burris (Eds.), Battelle Press. Columbus (Ohio), 1995.

(17) BRIERLEY, C.L. Geomicrobiol. J., 8, 1990: 201-223.

(18) Anderson, T.A. y CoATS, J.R. Bioremediation through Rhizosphere Technology, AMS, Washington, 1994.

(19) Bolton Jr., H., Fredrickson, J.K. y Elliot, L.F. Soil Microbial Ecology, F.B. Metting Jr. (Ed.), Marcel Dekker Inc., Nueva York, 1992.

(20) Cunningham, S.D., Berti, W.R. y Huang, J.W., Bioremediation of Inorganics. R.E. Hinchee, J.L. Means y D.R. Burris (Eds.), Battelle Press. Columbus (Ohio), 1995.

(21) Brown, S.L., Chaney, R.L., Angle, J.S. y Baker, A.J.M. J. Environ. Qual., 23, 1994: 1151-1157.

(22) Brown, S.L., Chaney, R.L., Angle, J.S. y Baker, A.J.M. Environ. Sci. Technol., 29, 1995: 1581-1585.
(23) Brown, S.L., Chaney, R.L., Angle, J.S. y Baker, A.J.M. Soil Sci. Soc. Am. J., 59, 1995: 125-133.

(24) Kumar, P.B.A.N., Dushenkov, V., Motto, H. y Raskin, I. Environ. Sci. Technol., 29, 1995: 1232-1238.

(25) Dushenkov, V., Kumar, P.B.A.N., Motto, H. y Raskin, I. Environ. Sci. Technol., 29, 1995: 1239-1245.

(26) KELLY, R.J. y GueRIN, T.F. Bioremediation of Inorganics. R.E. Hinchee, J.L. Means y D.R. Burris (Eds.), Battelle Press. Columbus (Ohio), 1995.

(27) Cornish, J.E., Goldberg, W.C., Levine, R.S. y BENEMANN, J.R. Bioremediation of Inorganics. R.E. Hinchee, J.L. Means y D.R. Burris (Eds.), Battelle Press. Columbus (Ohio), 1995.

(28) Cunningham, S.D. y Berti, W.R. In Vitro Cellular Dev., 20P, 1993, 207-212

(29) GADD, G.M. FEMS Microbiol. Rev., 11, 1993: 297-316.

(30) McCarty, S.L., Chasteen, T.G., Stalder, V. y BACHOFEN, R. Bioremediation of Inorganics. R.E. Hinchee, J.L. Means y D.R. Burris (Eds.), Battelle Press. Columbus (Ohio), 1995.

(31) Bachofen, R., Birch, L., Buchs, U., Ferloni, P., Flynn, I., Jud, G., TAhedl, H. y Chasteen, T.G. Bioremediation of Inorganics. R.E. Hinchee, J.L. Means y D.R. Burris (Eds.), Battelle Press. Columbus (Ohio), 1995.

(32) Saouter, E., Turner, R. y Barkay, T. Emerging Technology for Bioremediation of Metals, J.L. Means y R.E. Hinchee (Eds.), Lewis Pub., Boca Raton (Florida), 1994.

(33) Harwood-Sears, V. y Gordon, A.S. Appl. Environ. Microbiol., 56, 1990: 1327-1332.

(34) Schreiber, D.R., Millero, F.J. y Gordon, A.S. Marine Chem., 28, 1990: 275-284.

(35) Lauff, J.J., Steele, D.B., Coogan, A.L. y Breitfeller, J.M. Appl. Environ. Microbiol., 56, 1990: 3346-3353.

(36) Nörtmann, B. Appl. Environ. Microbiol., 58, 1992: 671676.

(37) Bolton Jr., H., Plymale, A.E. y Girvin, D.C. Abstracts of the 95th General Meeting of the ASM, 1995: 420.

(38) Silva-Avalos, J., Richmond, M.G., Nagappan, O. y KunZ, D.A. Appl. Environ. Microbiol., 56, 1990: 3664 3670.

(39) Finnegan, I., Toerien, S., Авbot, L., Smith, F. y Raubenheimer, H.G. Appl. Microbiol. Biotech., 36, 1991 142-144.

(40) Aronstein, B.N., Maka, A. y Srivastava, V.J. Appl. Microbiol. Biotech., 40, 1994: 700-707.

(41) Aronstein, B.N., Lawal, R.A. y MaKa, A. Environ. Toxic. Chem., 13, 1994: 1719-1726.

(42) Aronstein, B.N., Paterek, J.R., Rice, L.E. y SRIVAstaVA, V.J. Bioremediation of Inorganics. R.E. Hinchee, J.L. Means y D.R. Burris (Eds.), Battelle Press. Columbus (Ohio), 1995.

(43) Nagle, N.J., Rivard, C.J., Mohagheghi, A. y PhILIPPIDIS, G. Bioremediation of Inorganics. R.E. Hinchee, J.L. Means y D.R. Burris (Eds.), Battelle Press. Columbus (Ohio), 1995.

(44) Lovley, D.R. Adv. Agron., 54, 1994: 175-231.

(45) Rusin, P.A., Quintana, L., Brainard, R. Strietelmeier, B.A., Tait, C.D., Ekberg, S.A., Palmer, P.D., Newton, T.W. y Clark, D.L. Environ. Sci. Technol., 28, 1994: 1686-1690.

(46) Barkay, T., Turner, R.R., Vanderbrook, A. y Liebert, C., Microb. Ecol. 21, 1991: 151-161. 
(47) Silver, S. y Walderhaug, M. Microbiol. Rev., 56, 1992 195-228.

(48) Heinz, G.H., Pendelton, G.W., Krynitsky, A.J. y Gold, L.G. Arch. Environ. Contam. Toxicol., 19 (3), 1990: 374379.

(49) KIPPS, J.L. Emerging Technology for Bioremediation of Metals, J.L. Means y R.E. Hinchee (Eds.), Lewis Pub., Boca Raton (Florida), 1994.

(50) Owens, L.P., Kovac, K.C., KipPs, J.A.L. y Hayes, W.J. Bioremediation of Inorganics. R.E. Hinchee, J.L. Means y D.R. Burris (Eds.), Battelle Press. Columbus (Ohio), 1995.

(51) Lamb, B.E., Gold, A.J., LoOmIS, G.W. y McKiel, G.C. Trans. ASAE, 33 (2), 1990: 525-531.

(52) Graydon, J.W., OAKley, S.M., Reed, B.H. y Ball, H.L. Bioremediation of Inorganics. R.E. Hinchee, J.L. Means y D.R. Burris (Eds.), Battelle Press. Columbus (Ohio), 1995.

(53) Schmidt, G.C. y Ballew, M.B. Bioremediation of Inorganics. R.E. Hinchee, J.L. Means y D.R. Burris (Eds.), Battelle Press. Columbus (Ohio), 1995.

(54) Machemer, S.D., Reynolds, J.S., Laudon, L.S. y WildEMAN, T.R. Appl. Geochem., 8, 1993: 587-603.
(55) Wildeman, T.R., UpdegrafF, D.M., Reynolds, J.S. y BoLIS, J.L. Emerging Technology for Bioremediation of Metals, J.L. Means y R.E. Hinchee (Eds.), Lewis Pub., Boca Raton (Florida), 1994.

(56) Wildeman, T.R., Gusek, J., Cevaal, J., Whiting, K. y SCheuering, J. Bioremediation of Inorganics. R.E. Hinchee, J.L. Means y D.R. Burris (Eds.), Battelle Press. Columbus (Ohio), 1995.

(57) Farmer, G.H., UpdegrafF, D.M., Radehaus, P.M. y BATES, E.R. Bioremediation of Inorganics. R.E. Hinchee, J.L. Means y D.R. Burris (Eds.), Battelle Press. Columbus (Ohio), 1995.

(58) Tuovinen, O.H. Microbial Mineral Recovery, H.L. Ehrlich y C.L. Brierley (Eds.), McGraw-Hill Pub. Co., NuevaYork, 1990.

(59) Gupta, C.K. y Mukherjee, T.K. Hydrometallurgy in Extraction Processes, Vol.I, CRC Press, Boca Raton (Florida), 1990.

(60) Marsden, J. y House, I. The Chemistry of Gold Extraction, Ellis Horwood Ltd. Londres, 1992. 